\title{
Corynebacterium propinquum
}

National Cancer Institute

\section{Source}

National Cancer Institute. Corynebacterium propinquum. NCI Thesaurus. Code C86319.

A species of aerobic or facultatively anaerobic, Gram-positive, coryneform, pleomorphic bacilli assigned to the phylum Actinobacteria. This species is nonmotile, non-spore forming, catalase positive, oxidase negative, non-acid fast, non-lipophilic, reduces nitrate, does not hydrolyze urea, starch, or gelatin, and does not ferment glucose, sucrose, or maltose. C. propinquum is commensal to the oropharyngeal flora. 